\title{
Contact conductance of rough surfaces composed of modified RMD patches
}

\author{
M. Paggi ${ }^{\mathrm{a}, *, 1}$, J.R. Barber ${ }^{\mathrm{b}}$ \\ ${ }^{a}$ Leibniz Universität Hannover, Institut für Kontinuumsmechanik, Appelstraße 11, 30167 Hannover, Germany \\ ${ }^{\mathrm{b}}$ University of Michigan, Department of Mechanical Engineering, 2350 Hayward Street, Ann Arbor, MI, United States
}

\section{A R T I C L E I N F O}

\section{Article history:}

Received 15 December 2010

Received in revised form 1 June 2011

Accepted 3 June 2011

Available online 2 July 2011

\section{Keywords:}

Contact conductance

Contact stiffness

Fractal surfaces

Roughness

Waviness

Numerical methods

\begin{abstract}
A B S T R A C T
The dependence of the contact conductance of self-affine rough surfaces on the applied pressure is studied using the electric-mechanical analogy which relates the contact conductance to the normal stiffness. According to dimensional analysis arguments, an efficient dimensionless formulation is proposed which minimizes the number of dimensionless variables governing the phenomenon. Assuming incomplete similarity in the dimensionless pressure, a power-law dependence between contact conductance and mean pressure is proposed. This is confirmed by earlier semi-empirical correlations that are recovered as special cases of the proposed formulation. To compute the exponent $\beta$ of the power-law, and relate it to the morphological properties of the surfaces, we numerically test self-affine rough surfaces composed of random midpoint displacement (RMD) patches. Such patches are generated using a modified RMD algorithm in order to decouple the effect of the long wavelength cut-off from that due to microscale roughness. Numerical results show that the long wavelength cut-off has an important effect on the contact conductance, whereas the sampling interval and the fractal dimension are less important. The effect of elastic interaction between asperities has also been quantified and it significantly influences the predicted power-law exponent $\beta$.
\end{abstract}

(c) 2011 Elsevier Ltd. All rights reserved.

\section{Introduction}

The estimation of electric and thermal contact conductances across rough boundaries is a problem of paramount importance in engineering applications. Due to the roughness of contact surfaces, the real contact area is only a small percentage of the nominal one, significantly reducing the expected contact conductance. In the field of electronic packaging, the conductance between devices and dissipators must be increased as much as possible in order to maintain low operating temperatures. This would suggest the use of large contact areas to enhance dissipation, which is however a trend opposite to the need of miniaturization.

In this context, a pioneering textbook on the conductance of surfaces in contact is due to Holm [1]. More recently, in the 1970 s, a great attention has been devoted to the experimental computation of the thermal contact conductance of rough surfaces, leading to a series of publications by the research group coordinated by Yovanovich [2-8]. In these papers, semi-empirical correlations of power-law type were proposed:

$C=k p^{\beta}$

\footnotetext{
* Corresponding author. Tel.: +49 511762 3220/+39 0115644910.

E-mail addresses: paggi@ikm.uni-hannover.de, marco.paggi@polito.it (M. Paggi), jbarber@umich.edu (J.R. Barber).

${ }^{1}$ On leave from Politecnico di Torino, Italy.
}

where $C$ is the contact conductance, $p$ is the applied pressure, and the exponent $\beta$ was found to be somewhat lower than unity. In this respect, however, contradictory results appear in the literature. Some authors reported much lower values of $\beta$ in the range $0.5-0.6$, as recently recalled in [9]. In the framework of micromechanical contact theories, Greenwood and Williamson [10] proposed a linear dependence between contact conductance and pressure in case of an exponential distribution of asperity heights. With a more detailed description of asperity heights and curvatures according to the random process theory, Bush and Gibson [11] derived a correlation with an exponent $\beta=0.89$. Very recently, Persson [12] established a new contact model inspired by diffusion processes, suggesting again $\beta=1$, as in the Greenwood and Williamson theory [10]. On the other hand, previous numerical studies considering 2D Weierstrass profiles [9] suggested a best-fitting formula with an exponent $\beta$ larger than unity and dependent on the fractal dimension of the rough surface.

In the present study we investigate the relation between contact conductance and applied pressure using dimensional analysis considerations. By applying Buckingham's $\Pi$ theorem and incomplete similarity concepts, we derive a general relationship between dimensionless specific conductance and dimensionless pressure. Using such a relationship, we recover most of the previous semiempirical correlations available in the literature. Then, in order to estimate the power-law exponent $\beta$ and understand its dependencies, we perform numerical contact simulations on rough surfaces 


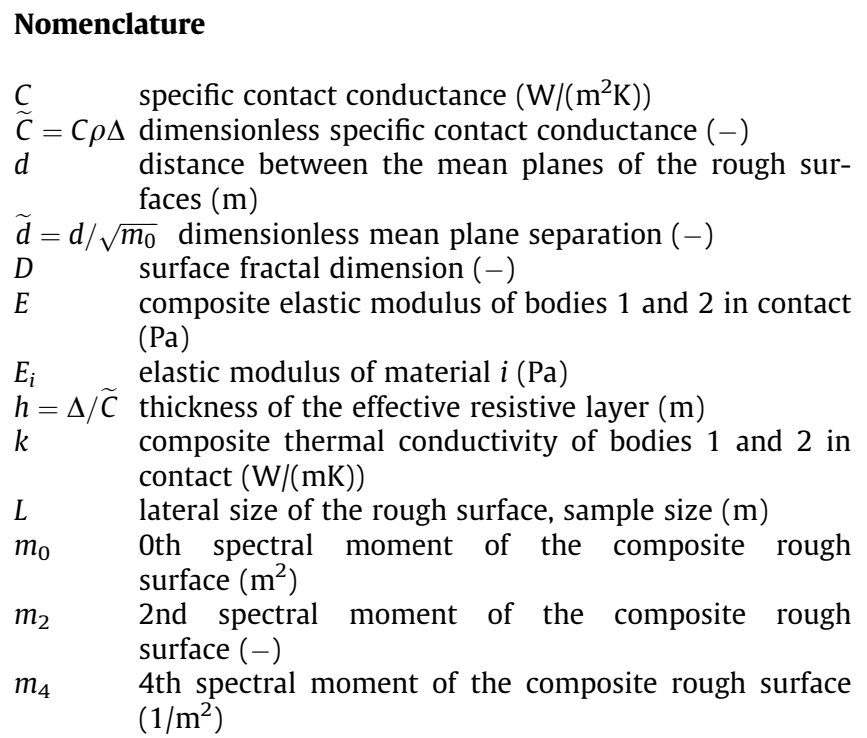

$p \quad$ nominal contact pressure (Pa)

$\widetilde{p}=p \Delta /\left(E \sqrt{m_{0}}\right)$ dimensionless pressure $(-)$

$w \quad$ contact compliance $(\mathrm{m})$

Greek symbol

$\alpha=m_{0} m_{4} / m_{2}^{2}$ bandwidth parameter $(-)$

$\beta \quad$ power-law exponent $(-)$

$\delta \quad$ sampling interval (m)

$\Delta \quad$ lateral size of a single RMD patch $(\mathrm{m})$

$\lambda \quad$ scalar multiplier $(-)$

$v_{i} \quad$ Poisson's ratio of material $i(-)$

$\rho \quad$ composite resistivity of bodies 1 and 2 in contact $(\mathrm{mK} / \mathrm{W})$

$\sigma_{i}^{2} \quad$ variance of the $i$ th random number generation of the RMD method $\left(\mathrm{m}^{2}\right)$

$\Phi, \Phi_{1}, \Phi_{2}$ dimensionless functions (-) composed of modified RMD patches. More specifically, the random midpoint displacement (RMD) generation algorithm is modified in order to generate surfaces with minimized waviness effects. This issue is particularly important for the contact conductance, which is primarily governed by the coarse scale topological features of the rough surface [13]. Applying the electric-mechanical analogy derived in [13], the specific contact conductance is determined via the derivative of the pressure vs. separation curve (incremental contact stiffness). Detailed numerical results will show the effect of the principal dimensionless numbers on the power-law exponent $\beta$. Specific attention will be devoted to the role played by elastic interaction between asperities, an important aspect neglected in previous investigations.

\section{A dimensional analysis approach to the scaling of contact conductance}

The specific contact conductance of rough surfaces, or conductance per unit area $C$, can be correlated to the the derivative of the force-indentation curve, i.e., the incremental stiffness, according to the electrical-mechanical analogy established in [13]. In formulae:

$C=-\frac{2}{\rho E} \frac{\mathrm{d} p}{\mathrm{~d} d}$

where $p$ is the nominal applied pressure and $d$ is the mean plane separation between the rough surfaces. The parameters $E$ and $\rho$ are the composite elastic modulus and resistivity of bodies 1 and 2 in contact:

$E=\left(\frac{1-v_{1}^{2}}{E_{1}}+\frac{1-v_{2}^{2}}{E_{2}}\right)^{-1}$,
$\rho=\rho_{1}+\rho_{2}$.

where $E_{i}, v_{i}, \rho_{i}(i=1,2)$ are Young's modulus, Poisson's ratio and resistivity respectively of the two contacting bodies. The dependence of the contact conductance due to roughness on the applied pressure can therefore be investigated by focusing on the incremental stiffness of the interface. Here, it should be remarked that steady-state thermal and electric conduction through a given set of contact spots are mathematically analogous problems. Therefore,
Eq. (2) holds also for thermal conduction, provided that $\rho$ is replaced by $1 / k$, where $k$ is the composite thermal conductivity of bodies 1 and 2, see [2]. Radiation and convection through the air gaps are not considered here, although in some cases these phenomena are important. On the other hand, in electric conduction problems, these aspects are negligible and other phenomena as the presence of insulated surface films may be relevant [14].

Practical surfaces often exhibit quasi-fractal properties at the fine scale, but generally there exists a cut-off to the power spectral density at small wavenumbers (long wavelengths). If this is not the case (e.g. if the surface is fractal in the Weierstrass-Mandelbrot sense), there must nonetheless exist a largest length dimension representing the finite size of the nominal contact area. Since the coarser features of the surface profile are known to dominate the contact conductance $[13,15]$, the length scales defining these two processes are important parameters in the problem description. Here we shall characterize the long wavelength cut-off by the length parameter $\Delta$ and the finite dimensions of the nominal contact area by $L$. We therefore propose the following functional dependence for the incremental stiffness per unit area:

$\frac{\mathrm{d} p}{\mathrm{~d} d}=\frac{\mathrm{d} p}{\mathrm{~d} d}\left(E, p, m_{0}, m_{2}, m_{4}, \delta, L, \Delta, D\right)$,

where $m_{0}, m_{2}$ and $m_{4}$ are the spectral moments of the rough surface [16], $D$ is the surface fractal dimension, and $\delta$ is the sampling interval. Notice incidentally that most theoretical studies of contact conductance tacitly assume that the effect of surface roughness can be decoupled from that of the macroscale conduction problem - in other words, that the effect of surface roughness is to add a nominal pressure-dependent additional resistance between the macroscopic contacting bodies. However, this decoupling of scales is justified only if $\Delta \ll L$.

The number of independent parameters in Eq. (4) can be reduced by applying the Buckingham's $\Pi$ theorem [17]. Most authors use the RMS roughness $\sqrt{m_{0}}$ to normalize the length parameters $[9,18]$, but here we shall show that when the above scale separation is possible, a greater reduction in the number of parameters is achieved by normalizing with respect to the long wavelength cut-off $\Delta$. We therefore write

$\frac{\Delta}{E} \frac{\mathrm{d} p}{\mathrm{~d} d}=-\Phi\left(\frac{p}{E}, \alpha, \frac{\sqrt{m_{0}}}{\Delta}, \frac{\delta}{\Delta}, \frac{L}{\Delta}, D\right)$, 
where the bandwidth parameter $\alpha=m_{0} m_{4} / m_{2}^{2}$ has been introduced. This parameter is dependent on the fractal dimension and on the resolution. For $\delta \ll \Delta$, the following approximation holds $[19,20](2<D<3)$ :

$\alpha \cong \frac{(D-2)^{2}}{(D-1)(3-D)}\left(\frac{\delta}{\Delta}\right)^{2(D-3)}$

Therefore, $\alpha$ will be dropped from the dependencies in (5), retaining only $D, \Delta$ and $\delta$ in the subsequent analysis. We can also use (2) to define the dimensionless specific contact conductance $\widetilde{C}$ as

$\widetilde{C} \equiv C \rho \Delta=-\frac{2 \Delta}{E} \frac{\mathrm{d} p}{\mathrm{~d} d}=2 \Phi\left(\frac{p}{E}, \alpha, \frac{\sqrt{m_{0}}}{\Delta}, \frac{\delta}{\Delta}, \frac{L}{\Delta}, D\right)$.

An alternative description of the contact conductance can be obtained by noting that the imperfect contact at the interface is equivalent to the interposition of a fictitious layer of the same material whose thickness $h$ is given by $\rho h=1 / C$. Normalizing this thickness with respect to $\Delta$, we then have

$\frac{h}{\Delta}=\frac{1}{\widetilde{C}}$.

Now consider the effect of holding $D, \delta, L$, and $\Delta$ constant and just rescaling $\sqrt{m_{0}}$ to $\lambda \sqrt{m_{0}}$, where $\lambda$ is a scalar multiplier. The contact problem is nonlinear, but the underlying elastic field is linear. Suppose that, at a given value of nominal pressure $p$, the actual pressure distribution is $p(x, y)$ and the actual contact area is $(x, y) \in \mathcal{A}$. It follows that the contact pressure distribution $\lambda p(x, y)$ acting over the same area $\mathcal{A}$ will produce displacements scaled in the same ratio $\lambda$ with respect to the original ones. These will be exactly what is needed to establish the contact area $\mathcal{A}$ when the roughness is characterized by $\lambda \sqrt{m_{0}}, D, \delta, L, \Delta$. Furthermore, the contact conductance depends only $\mathcal{A}$ and hence we have

$\Phi\left(\frac{\lambda p}{E}, \frac{\lambda \sqrt{m_{0}}}{\Delta}, \frac{\delta}{\Delta}, \frac{L}{\Delta}, D\right)=\Phi\left(\frac{p}{E}, \frac{\sqrt{m_{0}}}{\Delta}, \frac{\delta}{\Delta}, \frac{L}{\Delta}, D\right)$

for all scalar multipliers $\lambda$. Now suppose we define a new dimensionless parameter $p \Delta /\left(E \sqrt{m_{0}}\right)$ and use this to characterize the nominal pressure $p$. Eq. (7) will be modified to

$\widetilde{C}=\Phi_{1}\left(\frac{p \Delta}{E \sqrt{m_{0}}}, \frac{\sqrt{m_{0}}}{\Delta}, \frac{\delta}{\Delta}, \frac{L}{\Delta}, D\right)$

and due to the result in (9) we have

$\Phi_{1}\left(\frac{p \Delta}{E \sqrt{m_{0}}}, \frac{\lambda \sqrt{m_{0}}}{\Delta}, \frac{\delta}{\Delta}, \frac{L}{\Delta}, D\right)=\Phi_{1}\left(\frac{p \Delta}{E \sqrt{m_{0}}}, \frac{\sqrt{m_{0}}}{\Delta}, \frac{\delta}{\Delta}, \frac{L}{\Delta}, D\right)$

for all $\lambda$, showing that the new function $\Phi_{1}$ is independent of the dimensionless parameter $\sqrt{m_{0}} / \Delta$. We can therefore drop it from the functional dependence, obtaining

$\widetilde{C}=\Phi_{1}\left(\frac{p \Delta}{E \sqrt{m_{0}}}, \frac{\delta}{\Delta}, \frac{L}{\Delta}, D\right)$,

where the number of dimensionless parameters has been reduced from five to four.

At this point, it is interesting to note that the semi-empirical power-law correlations of type (1) can be recovered by dimensional analysis in case of incomplete similarity in the dimensionless number $p \Delta /\left(E \sqrt{m_{0}}\right)$. This condition usually applies in physical systems that are in an intermediate situation between two limit conditions [21]. For very low pressures, a deviation from the power-law behavior has been experimentally observed in [3]. This is attributed to the truncation of the asperity height distribution function of tested samples, which leads to an enhancement of thermal contact conductance at light contact pressures as compared to that predicted by a power-law equation. For typical values of the dimensionless truncation $d_{\max } / \sqrt{m_{0}}$ ranging from 3.5 to 4.5 , a deviation from the power-law scaling was experimentally ob- served for $p / E \lesssim 1 \times 10^{-5}$. Regarding the upper threshold, this may correspond to very high pressures, when the asperities merge with each other and create large contact clusters. This limit situation is however not reached in most of the cases, since we usually explore mean plane separations $d / \sqrt{m_{0}}>0$. In Section 6 , we shall perform contact simulations with $d / \sqrt{m_{0}}$ ranging from $d_{\max } / \sqrt{m_{0}}$ to zero, exploring at least four orders of magnitude of variation in the dimensionless contact pressure.

Assuming incomplete similarity in $p \Delta /\left(E \sqrt{m_{0}}\right)$, we can postulate a power-law dependence on this dimensionless parameter, giving

$\widetilde{C}=\left(\frac{p \Delta}{E \sqrt{m_{0}}}\right)^{\beta} \Phi_{2}\left(\frac{\delta}{\Delta}, \frac{L}{\Delta}, D\right)$,

where $\beta$ is the incomplete similarity exponent, which may depend on the other dimensionless numbers in parenthesis and can only be obtained from real or numerical experiments. The function $\Phi_{2}$ is a new dimensionless function of the remaining dimensionless parameters.

It is interesting to note that Eq. (13) is consistent with most of the semi-empirical correlations published in the literature for the thermal contact conductance of rough surfaces. Such correlations suggested power-law dependencies between the contact conductance and the applied pressure as in Eq. (1), with coefficients of proportionality depending on various statistical parameters. In the following, they are rewritten by modifying the original notation to make it consistent with the present formulation. In doing so, all the statistical parameters are reduced to the moments of the power spectral density and their combination $\alpha$ by using the equations in [20] given by random process theory. This enables us to compare the various correlations directly. Then, the moments $m_{0}, m_{2}, m_{4}$ and $\alpha$ are expressed in terms of the long wavelength cut-off $\Delta$, the sampling interval $\delta$, and the fractal dimension $D$, using again the relationships in [20]. This last approximation holds for self-affine rough surfaces with power spectral density of power-law type (fractal surfaces) and for $\delta \ll \Delta$.

Mikic [22] proposed a power-law correlation between conductance and pressure. Using an asperity model similar to that of Greenwood and Williamson [10], he finally fitted experimental results obtaining:

$$
\begin{aligned}
\widetilde{C} & =2.18\left(\frac{\sqrt{m_{0}}}{\Delta}\right)^{-0.06} m_{2}^{0.03}\left(\frac{p \Delta}{E \sqrt{m_{0}}}\right)^{0.94} \\
& \cong 2.43\left(\frac{3-D}{D-2}\right)^{0.03}\left(\frac{\delta}{\Delta}\right)^{0.06(2-D)}\left(\frac{p \Delta}{E \sqrt{m_{0}}}\right)^{0.94} .
\end{aligned}
$$

Comparing (14) with (13), we note that they are of the same form with $\beta=0.94$. Notice in particular the independence of the dimensionless ratio $\sqrt{m_{0}} / \Delta$. The Mikic model was subsequently reconsidered by the Yovanovich's group in a series of papers, proposing some variants. For instance, Blahey et al. [23] established the following correlation:

$$
\begin{aligned}
\widetilde{C} & =3.86\left(\frac{\sqrt{m_{0}}}{\Delta}\right)^{-0.07}\left(m_{2} \sqrt{\alpha}\right)^{0.035}\left(\frac{p \Delta}{E \sqrt{m_{0}}}\right)^{0.93} \\
& \cong 4.39 \frac{(D-1)^{-0.035}}{\sqrt{(D-1)(3-D)}}\left(\frac{\delta}{\Delta}\right)^{-0.035(D-1)}\left(\frac{p \Delta}{E \sqrt{m_{0}}}\right)^{0.93}
\end{aligned}
$$

which is also exactly of the form (13). Subsequently, Sridhar and Yovanovich [4] re-examined the Greenwood and Williamson theory [10] and proposed a correlation valid for $p / E>1 \times 10^{5}$ and for $5<\alpha<100$, where a slight dependence on the bandwidth parameter $\alpha$ suggested:

$\widetilde{C}=k_{S Y}\left(\frac{p}{E}\right)^{0.922 \alpha^{1 / 205.54}}$, 
where $k_{S Y}$ is a coefficient dependent on $\alpha$ (see [4] for more details). Such a dependence of the exponent $\beta$ on $\alpha$ is also plausible according to the previous dimensional analysis arguments, since $\beta$ may depend on the dimensionless numbers in Eq. (5).

Removing the assumption of a constant (average) radius of curvature for the asperities, Bush and Gibson [11] derived a correlation similar to the previous ones, but with a significantly lower exponent of $p / E$ :

$$
\begin{aligned}
\widetilde{C} & =1.38\left(\frac{\sqrt{m_{0}}}{\Delta}\right)^{-0.11}\left(m_{2} \sqrt{\alpha}\right)^{1 / 2} m_{2}^{-0.44}\left(\frac{p \Delta}{E \sqrt{m_{0}}}\right)^{0.89} \\
& \cong 1.69 \frac{(D-2)^{0.49}}{(D-1)^{0.25}(3-D)^{0.19}}\left(\frac{\delta}{\Delta}\right)^{0.78(D-2)-1}\left(\frac{p \Delta}{E \sqrt{m_{0}}}\right)^{0.89}
\end{aligned}
$$

which again is of the form of Eq. (13), this time with $\beta=0.89$. Notice that this model shows a significantly stronger dependence on the parameter $\delta / \Delta$.

Eq. (13) can be recast as an ordinary differential equation with separable variables, in order to determine the relationship between pressure and separation. We obtain

$\frac{\mathrm{d} \tilde{p}}{\tilde{p}^{\beta}}=-\frac{\Phi_{2}}{2} \mathrm{~d} \tilde{d}$

where we define the dimensionless variables

$\tilde{p}=\frac{p \Delta}{E \sqrt{m_{0}}} \tilde{d}=\frac{d}{\sqrt{m_{0}}}$.

If $\beta \neq 1$, this equation has the solution

$\frac{\tilde{p}^{1-\beta}}{1-\beta}=\frac{\Phi_{2}}{2}\left(\tilde{d}_{0}-\tilde{d}\right)$

where $\tilde{d}_{0}$ is a constant of integration. This is a power-law relationship between the applied pressure and the normal compliance associated with the asperities, $w_{a}=\tilde{d}_{0}-\tilde{d}$. Notice however that although the value $\tilde{d}=\tilde{d}_{0}$ corresponds to $\tilde{p}=0$, we cannot identify the constant $\tilde{d}_{0}$ with the highest point in the surface, since the power law behavior depends upon incomplete similarity which breaks down at very low pressures. Indeed, the highest point of the surface $\tilde{d}_{\text {max }}$ depends on extreme-value statistics of the surface and is likely to exhibit significant variance, making it unreliable as a parameter in a contact model.

For the special case $\beta=1$, the ODE (18) has the exponential solution:

$\tilde{p}=K \exp \left(-\frac{\Phi_{2} \tilde{d}}{2}\right)$,

where the dimensionless multiplying constant $K$ is related to the constant of integration and hence is undeterminate. A similar expression is of course obtained from the Greenwood and Williamson theory [10] when an exponential distribution of asperity heights is considered, leading to an expression of the form

$\tilde{p}=K_{\mathrm{GW}} \exp (-\tilde{d})$,

where the multiplying constant $K_{\mathrm{GW}}$ depends on the number of asperities per unit area and the summit radii. This agrees with the form (20) with $\Phi_{2}=2$.

Persson's recent theory [12] also suggests an exponential relation between $p$ and $d$ :

$p=\gamma E \exp \left(-d / d_{0}\right)$

where the coefficient $\gamma$ depends on the fractal dimension and on the length scales $\delta$ and $\Delta$, but was determined from a best fit. The constant $d_{0}$ was found to be of the order of magnitude of $\sqrt{m_{0}}$, implying $\Phi_{2}$ of order 2 in Eq. (20).
Finally, we remark that, in case of exponential relations between $\tilde{p}$ and $\tilde{d}$, a straightforward application of Eq. (13) leads to a linear dependence between $\widetilde{C}$ and $p / E$.

\section{Computation of the contact compliance related to the asperities}

If a finite flat rigid indenter were pressed into a perfectly flat half-space, there would be some elastic compliance and hence also a thermal resistance. Micromechanical contact theories, such as the model by Greenwood and Williamson [10] and Ciavarella et al. [24] attempt to describe the additional compliance or resistance associated with the surface roughness. When techniques based on the boundary element method [25] or the finite element method [26] are employed to solve a discrete version of the normal contact problem, the measured compliance is the sum of these two effects and it is necessary to subtract the compliance associated with the equivalent flat contact problem to extract the effect of the surface roughness.

If the nominal contact area comprises a square of dimensions $L \times L$, the mean specific conductance is [14]

$C=\frac{1}{0.868 \rho L}$,

implying that a mean nominal pressure $p$ will cause a displacement

$w_{0}=\frac{0.868 L p}{E}$.

The number 0.868 in Eq. (23) is a best-fitting coefficient estabished in [27] to fit the numerical results obtained with the boundary element method. If the motion of the rigid indenter is described by a normal displacement $w$, measured from the point of first contact $d_{\text {max }}$, we would normally expect to write $d=d_{\max }-w$, but in view of this discussion, the value appropriate to asperity deformation alone is

$d=d_{\max }-w+w_{0}$.

In other words, only the difference $w-w_{0}$ between the actual displacement and that which would occur with an equivalent flat surface should be used in computing the incremental stiffness associated with the surface roughness.

\section{Numerical generation of self-affine rough surfaces with minimized waviness effects}

When nonconforming contact takes place, as, e.g., when a rough sphere is pressed against a flat plane [28], the asperity compliance modifies the mean pressure on the macroscale and tends to reduce the difference between the singular pressure at the edges and the lower pressure in the middle. For very rough surfaces, the mean pressure would be approximately uniform. As investigated in $[6,29]$, the macroscopic surface curvature has an important effect on the overall contact conductance and only the microscale conductance defined by the effective layer thickness $h$ of Eq. (8) is independent of the surface profile. Therefore, decoupling the contribution due to microscale roughness from the overall contact conductance is particularly important, since this quantity is the only one that is not model-specific. If the roughness contribution is properly identified, then it is possible to translate the results into something that appears as an additional (mean) pressure dependent resistance at the interface. This result can then be used in other applications with more complex geometries involving waviness.

In order to decouple the contact resistance due to surface roughness from that due to the macroscopic geometry, we need 
the longest wavelength $\Delta$ associated with the roughness to be significantly smaller than the length dimension of the nominal contact area, which in the present instance requires that $L \gg \Delta$. To this aim, in this section we propose a modified method for the generation of fractal rough surfaces with minimized long wavenlength content.

Among the various methods to generate rough surfaces with selfaffine properties, we select the Random Midpoint Displacement (RMD) algorithm [30]. A random number generation, whose variance changes with scale, adds the requested details to the grid points of a progressively refined mesh. Few input parameters are necessary to generate these complex geometries [31]: the fractal dimension $D$, the initial variance of the random number extraction $\sigma_{0}^{2}$, and the nodal spacing, $\delta$. At the preliminary step, the heights of the four lattice corners are set equal to four random numbers (extracted from a Gaussian variable with zero mean and unit variance, see points labeled $j, s, o$ and $p$ in Fig. 1). The height of the central node $l$ is then computed as the average of the heights of the four corners, plus a value extracted from a Gaussian random variable with reduced variance $\sigma_{1}^{2}=\sigma_{0}^{2}(1 / \sqrt{2})^{3-D}$. The heights of the intermediate nodes $r, i$, $k$ and $q$ along the boundaries are determined as the interpolated height of the three neighboring nodes (for instance $p, l$ and $o$ for the node $r$ ), plus a value extracted from a Gaussian distribution with the new rescaled variance $\sigma_{2}^{2}=\sigma_{1}^{2}(1 / \sqrt{2})^{3-D}$. The recursive procedure is now repeated to each subcell with further divisions. Note that the heights of previous generation steps are no longer modified. As a result, the surfaces are such that the height field corresponding to the generation index $k$ embeds all the height-fields of the previous generation indices $(k-1, k-2, \ldots, 1)$.

Surfaces generated with the classical RMD algorithm have spectra with wavelengths that go all the way to the size of the tested specimen, and the results are quite sensitive to the detailed way they are constructed. Hence, they will generally exhibit an undesired random waviness whose effects will have a significant influence on the estimate of the contact conductance. This waviness is primarily due to the first step of the surface generation, where the heights of the four lattice corners are set equal to four random numbers. Further refinements of the height field just add more details on the initial geometry and therefore the power spectrum of the surface is no longer modified in the low frequency range.

In order to reduce such undesirable waviness effects of the single RMD patch, the four corner heights generated during the first step of the above RMD algorithm are set equal to zero. However, this modification is not exhaustive for the complete removal of waviness, which can still be induced by the generation of the subsequent heights. Thus, instead of generating a set of RMD samples differing from each other through the initial seed of the random number generator and testing each surface individually, we generate a single rough surface composed of $n \times n$ RMD patches. This permits us to have the longest wavelength associated with the roughness significantly smaller than the lateral size of the nominal contact area. Each patch has a lateral size $\Delta$, and the nominal surface size will be $L$. Therefore, for this generation method, the dimensionless number $L / \Delta$ is equal to $n$. The contact response of a patch of RMD surfaces should be, in some sense, equivalent to averaging over $n \times n$ different realizations of the smaller contact patches. However, clustering and contact spot distributions across patches might even dominate over the local asperity effects and can be relevant.

Hence, the RMD generation algorithm is repeated $n \times n$ times with different random seeds as input and the final surface is obtained by collecting all the individual RMD patches in a $n \times n$ grid. The height fields of the RMD patches are characterized by different average values which may be another source of waviness. For example, for a surface with $D=2.3, \delta=1 / 64$ and $n=8$, the difference between the average plane of each RMD patch, $z$, and the global average plane, $z^{*}$, divided by the global r.m.s. roughness, $\sqrt{m_{0}}$, varies approximately from +2 to -2 . This variability is particularly significant. To remove it, the height fields of each RMD patch are rescaled in order to match the average plane of the RMD patch which has the tallest asperity. In this way, when contact between the rough surface and a smooth plane is simulated, each asperity comes into contact in correspondence to the same separation from the mean plane as if the contact patches were tested separately from each other.

\section{Algorithm for the solution of the nonlinear contact problem}

The normal contact problem of rough surfaces generated with the modified RMD method described in the previous section is numerically solved using a discrete version of the Greenwood and Williamson contact theory, as firstly proposed by Ciavarella et al. [32]. Considering the contact between a rough surface and a smooth rigid plane, the solution procedure for each mean plane separation $d^{(j)}$ consists in the following steps:

(1) Asperities are identified from a 3D numerical scan as local summits, and their radii $R_{i}$ are measured.

(2) The initial contact domain is defined as in the original Greenwood and Williamson theory [10] by the set of asperities overlapping the smooth rigid plane, i.e., having $z_{i} \geqslant d^{(j)}$. This initial compliance of a generic asperity in contact, say $w_{i}=z_{i}-d^{(j)}$, is used to compute the contact radius $a_{i}$, its contact area $A_{i}$, and its load $P_{i}$ according to the Hertzian equations:

$$
\begin{aligned}
a_{i} & =\sqrt{R_{i} w_{i}}, \\
A_{i} & =\pi a_{i}^{2}, \\
P_{i} & =\frac{4}{3} \frac{E}{R_{i}} a_{i}^{3} .
\end{aligned}
$$
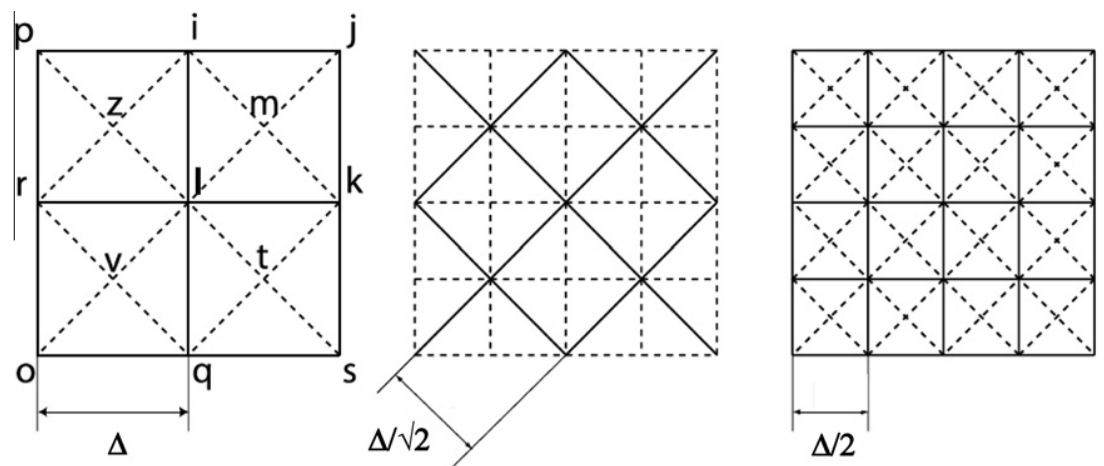

Fig. 1. Three successive RMD steps. 
The total contact area and total pressure without asperity interaction are then computed by summing all the asperity contributions.

(3) An iterative procedure is then applied to consider elastic interactions between asperities. In the generic $j$ th step, for a given distribution of contact dimensions $a_{i}^{(j)}$, the correction to the displacements $w_{i}^{(j)}$ due to the other asperities is the following [33]:

$$
\Delta w_{i}^{(j)}=\sum_{k=1, k \neq i}^{N} \frac{1}{\pi R_{k}}\left[\left(2 a_{k}^{2}-r_{i k}^{2}\right) \arcsin \frac{a_{k}}{r_{i k}}+a_{k} \sqrt{r_{i k}^{2}-a_{k}^{2}}\right],
$$

where the sum is extended to all the $N$ asperities in contact and $r_{i k}$ is the distance between the asperities $i$ and $k$. The compliances of the asperities are then updated as $w_{i}^{(j+1)}=w_{i}^{(j)}-\Delta w_{i}^{(j)}$. The contact condition $z_{i} \geqslant d_{i}$ is doublechecked. If satisfied, then the contact radius is updated as $a_{i}^{(j+1)}=\sqrt{R_{i} w_{i}^{(j+1)}}$, otherwise $a_{i}^{j+1}=0$. The procedure is repeated until the maximum relative error in the computed asperity displacements is less than the tolerance of $1 \times 10^{-12}$. This relative error is defined as $\max \left(\left|\Delta w_{i}^{(j)}\right| / w_{i}^{(j)}\right)$.

(4) The total real contact area $A_{r}$ and the total pressure with asperity interaction effects are finally computed by summing the individual asperity contributions. Then, we move to the next value of the mean plane separation and we repeat from the step (2).

Convergence of the numerical algorithm is quite fast, as illustrated in Fig. 2 for a generic mean plane separation. In the first four iterations, a quadratic convergence is achieved, since the error progresses as $1 \times 10^{0}, 1 \times 10^{-2}, 1 \times 10^{-4}, 1 \times 10^{-8}$. This implies that the number of accurate digits doubles from one iteration to the next. Afterwards, a superlinear convergence is preserved, until the achievement of the prescribed tolerance of $1 \times 10^{-12}$.

\section{Parametric analysis}

The relationships between normal contact stiffness and pressure and between pressure and compliance of self-affine rough surfaces are here investigated in reference to the self-affine surfaces composed of modified RMD patches. The normal contact problem is solved numerically according to the algorithm proposed in Section 5. In contrast to the semi-analytical solutions provided by micromechanical contact theories, the numerical approach used here permits us to analyze an actual representative surface topology (distribution of asperity heights and asperity curvatures) without making simplifying assumptions. Most importantly, it

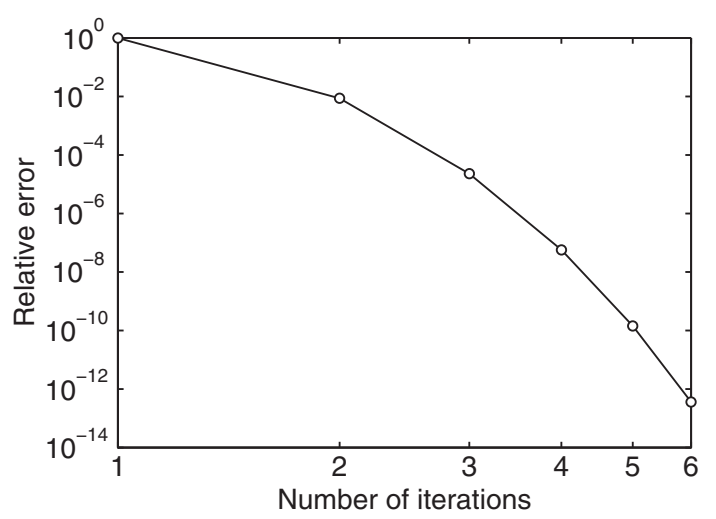

Fig. 2. Convergence of the numerical method. takes into account elastic interactions between asperities, a feature not considered in the studies that led to the correlations reported in Section 2.

Parametric analyses are performed by varying the dimensionless numbers $L / \Delta, \delta / \Delta$, and $D$. Starting with $L / \Delta$, we select $D=2.3, \delta / \Delta=1 / 64$, and we generate surfaces with $n \equiv L / \Delta=2,4$, and 8. Each tested surface is composed of $n \times n$ surface patches having the same lateral size $\Delta$. The dimensionless pressure vs. separation response of the rough surfaces is depicted in Fig. 3, with or without asperity interactions. The response of a single $(n=1)$ standard RMD patch is also superimposed with open circles for comparison. Neglecting asperity interaction (Fig. 3(a)), the curves tend to converge to the same pressure level, for very low separations. On the other hand, when asperity interaction is included, the various cases show quite distinct behavior. As a general trend, the higher the lateral size, the lower the asymptotic pressure. Notice that on this log-linear scale, a straight line would correspond to an exponential relation between pressure and separation, as suggested by the original Greenwood and Williamson contact theory with an exponential distribution of asperity heights, or in the recent Persson's theory. As it can be seen, the deviation from linearity is quite evident, especially when elastic interactions between asperities are taken into account.

The dimensionless specific conductance, computed via the derivative of the pressure-separation curves in Fig. 3, is plotted as a function of the dimensionless pressure in Fig. 4. The differentiation is carried out according to the forward finite difference scheme. The low regularity of the dimensionless pressure vs. separation curve for $\tilde{d} \sim 3$ in case of $L / \Delta=8$ is due to the presence of a small number of asperities in contact, which makes the results statistically not representative of the surface behavior. Reducing the separation $(\tilde{d}<3)$, more asperities come in contact and the curve becomes smoother.

Without interaction effects (Fig. 4(a)), the relation between dimensionless contact stiffness and applied pressure is well approximated by a power-law with exponent $\beta=0.82$. This exponent is more or less independent of $L / \Delta$. However, the results for a single standard RMD patch (empty dots, green in the online version) that does not possess the scale separation of the modified patches, would lead to a lower slope equal to 0.74 . When elastic interactions are considered (Fig. 4(b)), two regimes can be observed. For low contact pressures, $\tilde{p} \lesssim 1 \times 10^{-1}$, the contact stiffness is a power-law function of the pressure with an exponent $\beta \sim 0.72$ which is almost independent of $L / \Delta$. At higher pressures, the slope reduces, with $\beta \sim 0.46$. By increasing the ratio $L / \Delta$, the gap between the curves tends to diminish, indicating a convergence as theoretically expected. In the theoretical limit of $L / \Delta \rightarrow \infty$, the effect of the finite dimensions of the nominal contact area on the microscale resistance due to roughness should vanish.

Note that the maximum dimensionless pressure attained for the simulations in case of asperity interactions is significantly lower than that observed when elastic interactions are neglected. We remark here that all the simulations are performed up to a minimum dimensionless separation equal to $\widetilde{d}_{\min }=0$, which physically means that $50 \%$ of the asperities have been brought into contact. This separation is usually considered low enough to obtain a high pressure regime. The rough surface with $D=2.3, L / \Delta=2$ and $\delta / \Delta=1 / 64$ has also been tested up to $\tilde{d}_{\text {min }}=-2$, which corresponds to $98 \%$ of asperities in contact. The computing time drastically increases and the dimensionless pressure taking into account the elastic interactions changes from 0.9 to 2.5 , which is in any case less than the maximum pressure of $\tilde{p} \sim 10$ attained without interactions for $\tilde{d}_{\min }=0$. The transitional value of $\tilde{p} \sim 1 \times 10^{-1}$ for the pressure delimiting the two power-law regimes was fully confirmed.

Considering now the parameter $\delta / \Delta$, we select $D=2.3$ and $L / \Delta=2$. The RMD surfaces are generated with three different 


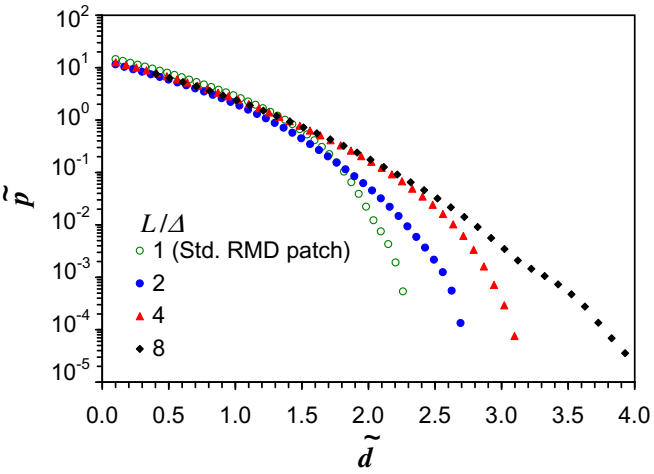

(a) Without asperity interaction

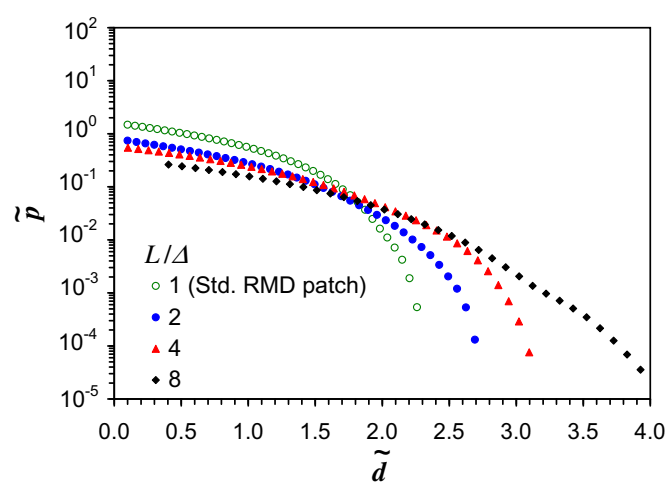

(b) With asperity interaction

Fig. 3. Effect of $L / \Delta$ on the dimensionless pressure vs. separation relation $(\delta / \Delta=1 / 64, D=2.3)$.

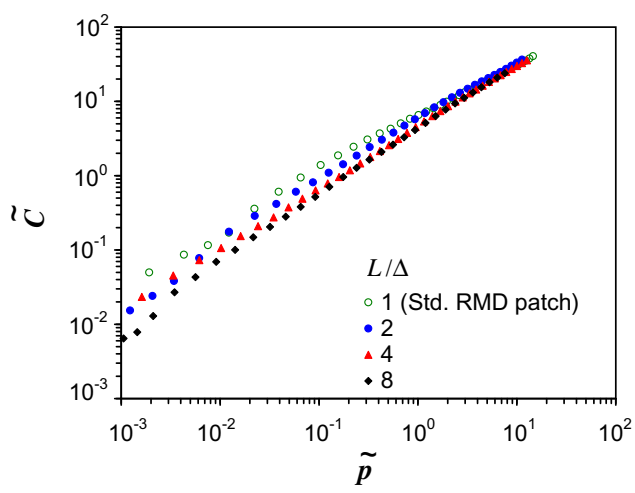

(a) Without asperity interaction

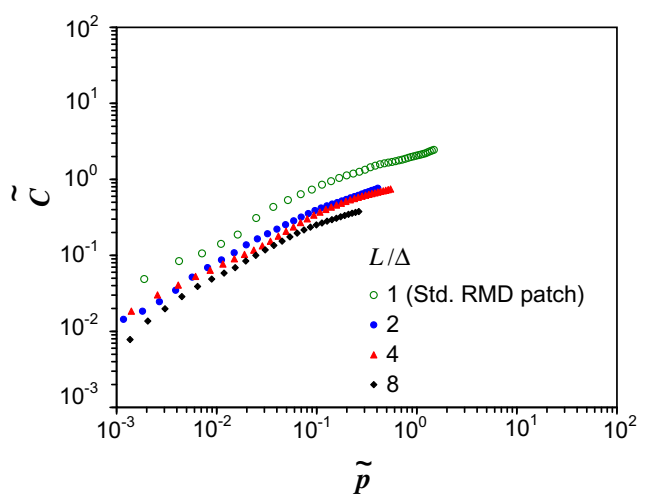

(b) With asperity interaction

Fig. 4. Effect of $L / \Delta$ on the dimensionless specific contact conductance $(\delta / \Delta=1 / 64, D=2.3)$.

resolutions, such that $\delta / \Delta$ is $1 / 32,1 / 64$, and $1 / 128$. The higher is $\delta /$ $\Delta$, the coarser is the surface. To give an idea of the computational complexity of the problem, the surface with the finest resolution presents nearly 20000 asperities in contact. As we know from the bounds theorem [13], the effect of shorter wavelengths is bounded and it reduces as we reduce the short wavelength cut-off $\delta$. Any dependence on this parameter has no physical significance, unless there is still such a dependence when we get down to length scales comparable with atomic dimensions, where fractality (and indeed continuum theory) must break down. The dimensionless specific contact conductance for these surfaces is plotted as a function of the dimensionless pressure in Fig. 5. Without interaction effects (Fig. 5(a)), the relation between dimensionless contact stiffness

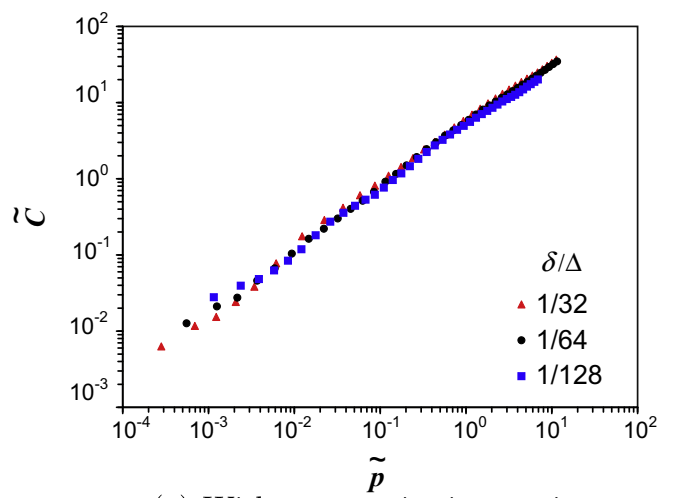

(a) Without asperity interaction and applied pressure is again well approximated by a power-law with exponent $\beta=0.81$. This exponent is independent of $\delta / \Delta$, as expected. When elastic interactions are considered (Fig. 5(b)), two regimes can be observed. For low contact pressures, $\tilde{p} \lesssim 1 \times 10^{-1}$, the contact stiffness is a power-law function of the pressure, with an exponent $\beta \sim 0.73$. Afterwards, the exponent is diminished and it is approximately equal to $\beta \sim 0.46$. Also in this case, no significant difference can be noticed in the three cases, confirming that the contact conductance due to roughness is mainly governed by the long wavelength cut-off.

Finally, we examine the effect of the fractal dimension, considering $L / \Delta=2, \delta / \Delta=1 / 64, D=2.3,2.5$ and 2.7. The pressure vs. compliance response of the rough surfaces is depicted in Fig. 6,

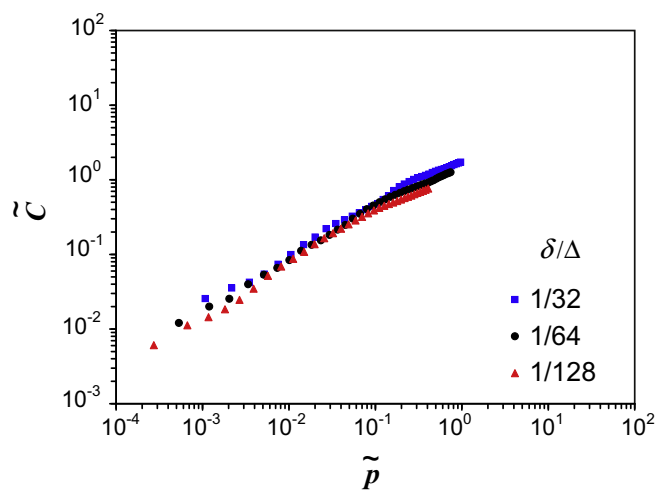

(b) With asperity interaction

Fig. 5. Effect of $\delta / \Delta$ on the dimensionless specific contact conductance $(L / \Delta=2, D=2.3)$. 


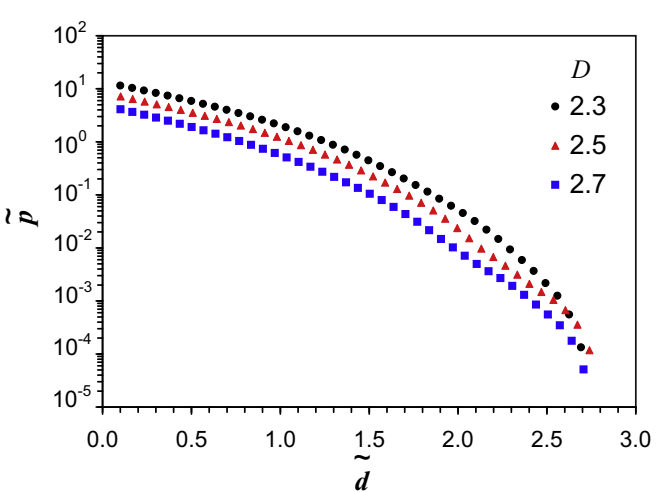

(a) Without asperity interaction

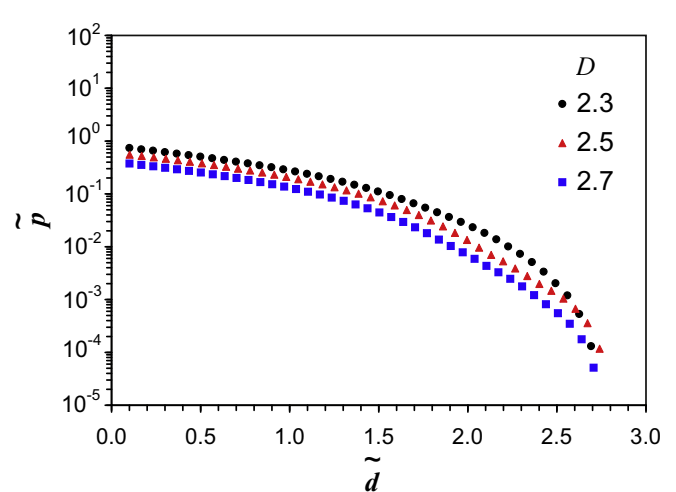

(b) With asperity interaction

Fig. 6. Effect of $D$ on the dimensionless pressure vs. separation.

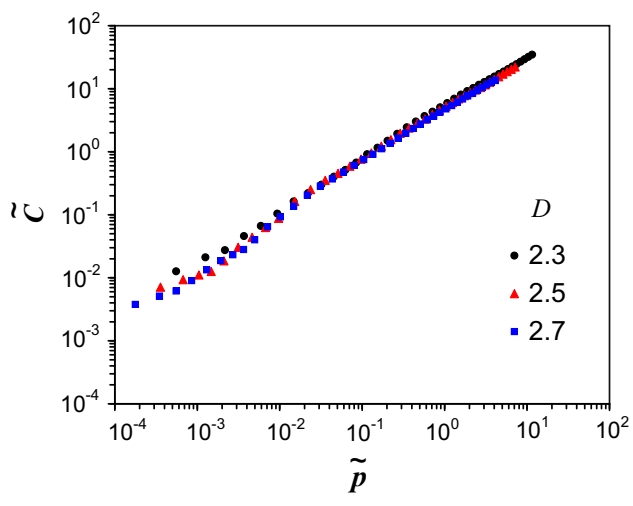

(a) Without asperity interaction

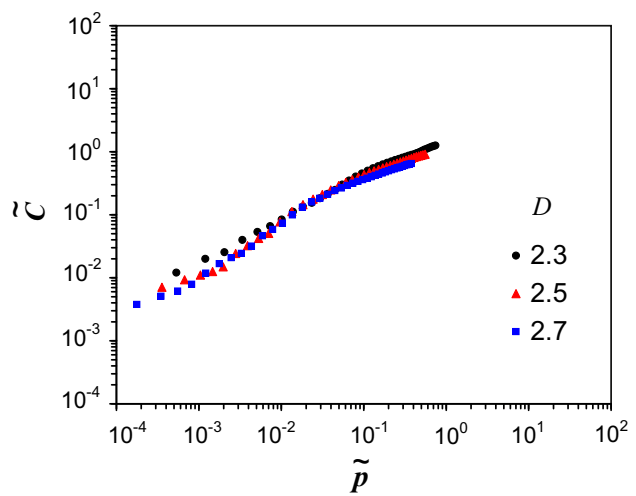

(b) With asperity interaction

Fig. 7. Effect of $D$ on the dimensionless specific contact conductance.

with or without asperity interaction effects. When asperity interactions are considered, the responses become very similar to each other, in the whole range of separations.

The dimensionless specific contact conductance vs. pressure is shown in Fig. 7. Without interaction effects (Fig. 7(a)), the exponent $\beta$ of the power-law approximation is 0.82 , almost independently of $D$. When elastic interactions are considered (Fig. 7(b)), again two different regimes can be noticed, depending on the pressure level. For $\tilde{p} \lesssim 1 \times 10^{-1}, \beta \sim 0.71$, whereas for $\tilde{p} \gtrsim 1 \times 10^{-1}$ we find $\beta \sim 0.48$.

\section{Conclusions}

In this paper, the dependence of the contact conductance of self-affine rough surfaces on the applied pressure has been investigated using the electric-mechanical analogy which relates the contact conductance to the normal stiffness. According to preliminary dimensional analysis arguments, an efficient dimensionless formulation has been proposed, reducing the number of dimensionless variables to four. They correspond to the dimensionless pressure, the dimensionless sampling interval, the dimensionless surface size, and the surface fractal dimension. Moreover, a power-law dependence on the dimensionless pressure has been postulated, based on considerations of incomplete similarity.

Using this formulation, it has been shown that most of the earlier semi-empirical correlations of power-law type can be recovered as special cases. Moreover, its integration permits us to determine the pressure vs. separation relation, which can be of either power-law or exponential type. The latter is obtained when $\beta=1$, as in the
Greenwood and Williamson theory with an exponential distribution of asperity heights and in the recent Persson's theory.

To compute the exponent $\beta$ of the power-law, and relate it to the morphological properties of the surfaces, we then performed a series of numerical tests on self-affine rough surfaces generated by the random midpoint displacement (RMD) method. Rough surfaces were obtained as a collection of modified RMD patches, in an attempt to minimize long wavelength effects and isolate the response due to roughness. The results show significant dependence on the ratio $L / \Delta$ between the nominal contact dimension $L$ and the long wavelength cut-off $\Delta$, but the results appear to converge as $L / \Delta$ is increased. By contrast, the power-law exponent $\beta$ is relatively insensitive to $L / \Delta$.

The short wavelength cutoff $\delta$ was found to have very little influence on the conductance, as theoretically predicted by the bounds theorem [13].

Finally, the effect of the fractal dimension has been investigated. As a general trend, when asperity interactions are neglected, we find $\beta \sim 0.82 \pm 0.01$. This value is not too different from $\beta=0.89$ analytically predicted by Bush and Gibson [11], who considered distributions of asperity heights and curvatures according to the random process theory. In our present computations, no assumptions on the distributions of asperities and curvatures are made, since the actual geometries of the rough surfaces are considered in the numerical simulations. When asperity interactions are included, the power-law exponent diminishes significantly relative to that computed in absence of elastic interactions. More specifically, we find two different regimes, depending on the pressure level. For low dimensionless contact pressures $\left(\tilde{p} \lesssim 1 \times 10^{-1}\right)$, which corresponds approximately to $p / E \lesssim 1 \times 10^{-3}$ for all the tested 
surfaces, we have $\beta \sim 0.72 \pm 0.01$. For higher pressure levels $(\tilde{p} \gtrsim$ $\left.1 \times 10^{-1}\right)$, the exponent is further reduced, having $\beta \sim 0.47 \pm 0.01$. These results may contribute to the understanding of the large scatter of results reported in the literature.

\section{Acknowledgements}

The first authors would like to thank the Alexander von Humboldt Foundation for supporting his research fellowship at the Institut für Kontinuumsmechanik, Leibniz Universität Hannover (Hannover, Germany) from February 1, 2010, to January 31, 2011.

\section{References}

[1] R. Holm, Electric Contact. Theory and Applications, Springer, Berlin, England, 1958.

[2] M.G. Cooper, B.B. Mikic, M.M. Yovanovich, Thermal contact conductance, Int. J. Heat Mass Transfer 12 (1968) 279-300.

[3] F.H. Milanez, M.M. Yovanovich, J.R. Culham, Effect of surface asperity truncation on thermal contact conductance, IEEE Trans. Compon. Packag. Technol. 26 (2003) 48-54.

[4] M.R. Sridhar, M.M. Yovanovich, Contact conductance correlations based on Greenwood and Williamson surface model, in: ASME National Heat Transfer Conference, Thermal Contact Resistance Session, Houston, Texas, ASME, 1996, pp. 1-11.

[5] M.R. Sridhar, M.M. Yovanovich, Elastoplastic contact conductance model for isotropic, conforming rough surfaces and comparison with experiments, J. Heat Transfer 118 (1996) 3-16.

[6] M. Bahrami, J.R. Culham, M.M. Yovanovich, G.E. Schneider, Thermal contact resistance of nonconforming rough surfaces, Part 2: Thermal model, J. Thermophys. Heat Transfer 18 (2004) 218-227.

[7] M.M. Yovanovich, A.A. Hegazy, J. De Vaal, Hardness distribution effects upon contact, gap and joint conductances, AIAA Paper, St. Louis, Missouri, (82-0887), 1982.

[8] M.M. Yovanovich, A.A. Hegazy, V.W. Antonetti, Experimental verification of contact conductance models based upon distributed surface micro-hardness, AIAA Paper, Reno, NV, (83-0532), 1983.

[9] M. Ciavarella, S. Dibello, G. Demelio, Conductance of rough random profiles, Int. J. Solids Struct. 45 (2008) 879-893.

[10] J.A. Greenwood, J.B.P. Williamson, Contact of nominally flat surfaces, Proc. Roy. Soc. Lond. Ser. A 295 (1966) 300-319.

[11] A.W. Bush, R.D. Gibson, A theoretical investigation of thermal contact conductance, Appl. Energy 5 (1979) 11-22.

[12] B. Lorenz, B.N.J. Persson, Interfacial separation between elastic solids with randomly rough surfaces: comparison of experiment with theory, J. Phys. Condens. Matter 21 (2008) 015003.
[13] J.R. Barber, Bounds on the electrical resistance between contacting elastic rough bodies, Proc. Roy. Soc. Lond. Ser. A 459 (2003) 53-66.

[14] L. Boyer, Contact resistance calculations: Generalizations of Greenwood's formula including interface films, IEEE, Trans. Comput. Packag. Technol. 24 (2001) 50-58.

[15] J.A. Greenwood, J.J. Wu, Surface roughness and contact: an apology, Meccanica 36 (2001) 617-630.

[16] P.R. Nayak, Random process model of rough surfaces, ASME J. Lubr. Technol. 93 (1971) 398-407.

[17] E. Buckingham, Model experiments and the form of empirical equations, ASME Trans. 37 (1915) 263-296.

[18] M.R. Sridhar, M.M. Yovanovich, Review of elastic and plastic contact conductance models: Comparison with experiments, J. Thermophys. Heat Transfer 8 (4) (1994) 633-640.

[19] R.S. Sayles, T.R. Thomas, The spatial representation of surface roughness by means of the structure function: a practical alternative to correlation, Wear 42 (1977) 263-276.

[20] G. Zavarise, M. Borri-Brunetto, M. Paggi, On the resolution dependence of micromechanical contact models, Wear 262 (2007) 42-54.

[21] G.I. Barenblatt, Scaling, Self-similarity and Intermediate Asymptotics, Cambridge University Press, Cambridge, 1996.

[22] B.B. Mikic, Thermal contact conductance: theoretical considerations, Int. J. Heat Mass Transfer 17 (1974) 205-214.

[23] A. Blahey, J.L. Tevaarwerk, M.M. Yovanovich, Contact conductance correlations of elastically deforming flat rough surfaces, AIAA Paper No. 80-1470 presented at the AIAA 5th Thermo-physics Conference, Snowmass, Colorado, 1980

[24] M. Ciavarella, J.A. Greenwood, M. Paggi, Inclusion of interaction in the Greenwood \& Williamson contact theory, Wear 265 (2008) 729-734.

[25] M. Borri-Brunetto, A. Carpinteri, B. Chiaia, Scaling phenomena due to fractal contact in concrete and rock fractures, Int. J. Fract. 95 (1999) 221-238.

[26] S. Hyun, L. Pei, J.-F. Molinari, M.O. Robbins, Finite-element analysis of contact between elastic self-affine surfaces, Phys. Rev. E 70 (2004) 026117.

[27] M. Nakamura, Constriction resistance of conducting spots by the boundary element method, IEEE Trans. Compon. Hybrids Manuf. Technol. 16 (1993) 339-343.

[28] J.A. Greenwood, J.H. Tripp, The elastic contact of rough spheres, J. Appl. Mech. 34 (1967) 153-159.

[29] M. Bahrami, J.R. Culham, M.M. Yovanovich, G.E. Schneider, Thermal contact resistance of nonconforming rough surfaces, Part 1: Contact mechanics model, J. Thermophys. Heat Transfer 18 (2004) 209-217.

[30] H.O. Peitgen, D. Saupe, The Science of Fractal Images, Springer-Verlag, New York, 1988.

[31] J. Feder, Fractals, Plenum Press, New York, 1988.

[32] M. Ciavarella, V. Delfine, G. Demelio, A "re-vitalized" Greenwood \& Williamson model of elastic contact between fractal surfaces, J. Mech. Phys. Solids 54 (2006) 2569-2591.

[33] K.L. Johnson, Contact Mechanics, Cambridge University Press, Cambridge, 1985. 